\title{
Intra-examiner and inter-examiner reliability in the determination of angular measurements of the wrist using a smartphone application
}

\author{
Talita Wassmuth ${ }^{1}$, Mariana Saori Barboza Sakata ${ }^{1}$, Andressa Morais de Paula ${ }^{1,}$ Wagner Menna Pereira ${ }^{2,}$ Luiz Alfredo Braun \\ Ferreira², Luciano Pavan Rossi ${ }^{1}$.
}

${ }^{1}$ Universidade Estadual do Centro-Oeste - UNICENTRO, Guarapuava (PR), Brazil, ${ }^{2}$ Centro Universitário Guairacá. Universidade Estadual do Centro-Oeste, Unicentro, Guarapuava (PR), Brazil.

\section{ABSTRACT:}

Background: With recent technological advances, goniometric applications have increasingly been used for the measurement of joint angles due to the greater practicality and accessibility. It is therefore important to determine the reliability of these applications. The evaluation of the wrist is important, as this joint is commonly affected by trauma that can lead to limitations of movement. Objective: the aim of the study was to determine the intra-examiner and inter-examiner reliability of the Goniometer $P R O^{\circ}$ smartphone application for measuring the range of motion of the wrist. Methods: A correlational, blind, cross-sectional study was conducted involving 43 healthy women (mean age: $20 \pm 2.3$ years) with no upper limb injuries. Both wrists were examined in each participant $(n=86)$. Active wrist flexion and extension were measured by three previously trained examiners using the smartphone application for the determination of inter-examiner reliability and a second evaluation was performed after 48 hours for the determination of intra-examiner reliability. Results: Excellent intra-examiner (ICC $>0.75$ ) and inter-examiner (ICC $>0.90$ ) reliability were found for both wrists. A very good correlation $(r>0.90 ; p<0.0001)$ was found for the intra-examiner evaluation of the left wrist and good correlations $(r>0.80 ; p<0.0001)$ were found for the right wrist and for the inter-examiner evaluations of both wrists. Good agreement was found for all measures, as demonstrated by the Bland-Altman analysis. Conclusions: The present data on intra-examiner and inter-examiner reliability of the Goniometer $P R O^{\odot}$ for the smartphone demonstrate that this goniometric application can be used as an effective tool for the evaluation of the range of motion of the wrist.

Keywords: Smartphone; Joint range of motion; Wrist; Reproducibility of measurements.

\section{BACKGROUND}

Applications for smartphones have been developed for the field of health and fitness, such as goniometric applications for the measurement of joint range of motion (ROM) ${ }^{(1)}$. These applications have advantages in terms of usability and involve fewer technical difficulties ${ }^{(2)}$.

The use of goniometric applicative facilitates accessibility and offers resources that a universal goniometer does not have, such as the recording of several data points and instantaneous recording with just the touch of a button, enabling the reading to be made with greater precision ${ }^{(3,4)}$. The evaluation of ROM is one of the main criteria used for the evaluation of functional limitations, the determination of diagnoses and treatment plans and the quantitative analysis of the effects of rehabilitation ${ }^{(5)}$. However, it is important to determine whether these applications are valid and reliable for use in the clinical setting and research ${ }^{(6,3,4)}$.

There are several methods for measuring ROM, from a visual inspection to the use of precise tools and estimates produced on a computer ${ }^{(5)}$. Although a visual inspection is likely sufficient for a single therapist examining a patient over time, this method has little inter-examiner reliability and a low measurement precision ${ }^{(7)}$. Therefore, the universal goniometer is the most widely used tool by physiotherapists in clinical practice for the evaluation of the ROM of joints ${ }^{(6)}$, with good intra-examiner reliability and clinical validity. Moreover, a strong correlation has been found between the universal goniometer and goniometric applications for smartphones, with minimal differences in angular measurements ${ }^{(7)}$.

Reliability regards the consistency of successive measurements of the same variable on the same individual under the same temporospatial conditions by different examiners. This concept involves characteristics such as coherence, stability, equivalence and homogeneity, making it one of the main criteria of the quality of a given instrument ${ }^{(8)}$. According to Bolarinwa (2015), reliability regards the results of a measurement and a method that can be reproduced. There are three aspects of reliability that should be considered: the equivalence, stability and homogeneity of the measure. These aspects play a role in guiding researchers regarding the appropriate reliability of a research instrument ${ }^{(9)}$.

The wrist is a complex, multi-directional joint that is susceptible to trauma and fractures, which can lead to limitations in $\mathrm{ROM}^{(10)}$. Therefore, the measurement of the ROM of the wrist with a valid, reliable instrument can assist therapists in quantifying movement limitations, monitoring the effectiveness of treatment and the progression of recovery as well as improving communication between therapists ${ }^{(11)}$. 
The high number of injuries to the wrist and the importance of this joint to activities of daily living justify the present investigation.

The aim of this study was to analyze intra-examiner and inter-examiner reliability regarding angle measurements of the wrist during flexion and extension using a goniometric smartphone application.

\section{METHODS \\ Participants}

A double-blind, cross-sectional study was conducted at the Physical Therapy Teaching Clinic of the Central West State University. The sample was composed of 43 healthy, young women (mean age: $20 \pm 2.3$ years; height: $164 \pm 7 \mathrm{~cm}$; body mass: $62 \pm 11.6 \mathrm{~kg}$; body mass index: $23 \pm 3.7 \mathrm{~kg} / \mathrm{m}^{2}$ ). Both wrists of each participant were examined $(n=86)$. The inclusion criteria were age 18 to 30 years, the female sex and no recent history of an upper limb injury. This study received approval from the ethics committee of the Central West State University, Brazil (certificate number: 3.407.073).

\section{Procedures and tools}

A pilot study was first conducted to familiarize the examiners with the joint measurement instruments and determine possible errors that may occur during the evaluations. Three trained examiners independently performed the evaluations for the determination of interexaminer reliability. After 48 hours, the examiners performed the evaluations a second time for the determination of intraexaminer reliability. The examiners were blinded to the measurements of the other examiners and were also unaware of their own measurements. A fourth researcher performed the reading of the measurements to minimize the possibility of measurement bias among the examiners.

Active flexion and extension of the wrist was performed, as recommended by Gehrmann ${ }^{(12)}$, since active wrist movements are fundamental to the performance of activities of daily living. The application used was the Goniometer Pro ${ }^{\odot}$ (https://itunes.apple.com/us/app/goniometer-

pro/id646925503?mt=8) for smartphone model Samsung Galaxy (Figure1).

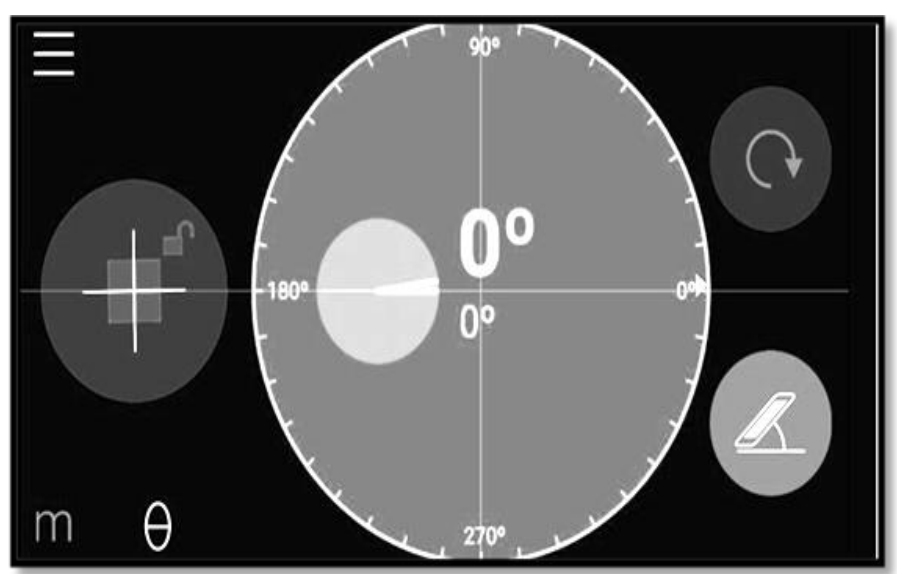

Figure 1. Readout display of joint angle using Goniometer Pro ${ }^{\odot}$ smartphone application.
The participant was seated with the shoulder abducted $30^{\circ}$, elbow flexed $30^{\circ}$ and forearm pronated. A Velcro ${ }^{\mathrm{TM}}$ strap was used to secure the distal third of the forearm to the support table (Figure 2).

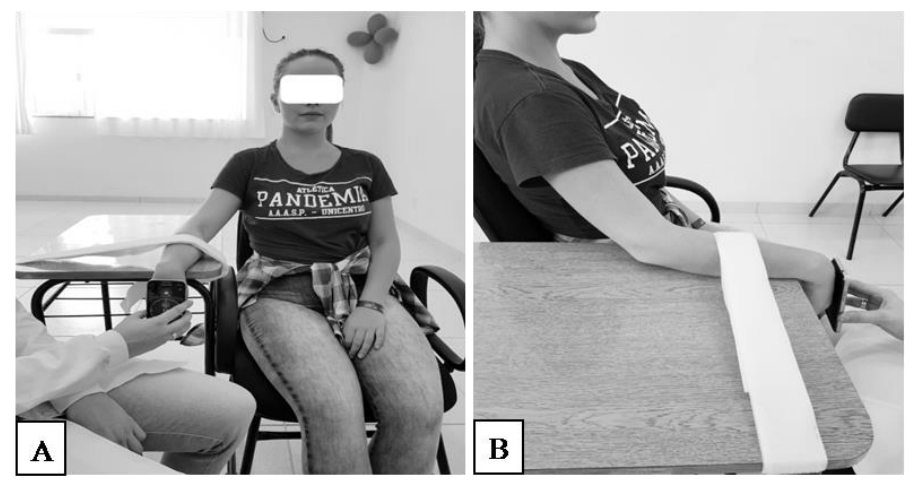

Figure 2. Positioning of participant and smartphone in forward (A) and side (B) views.

The examiner removed the protective sleeve of the smartphone and any adornment that could interfere with the evaluation. The device was positioned so that its posterior portion was in contact with the dorsal region of the participant's wrist ${ }^{(13)}$, as shown in Figure 2.

ROM of the wrist was measured three times for the active movements of flexion and extension. These movements are essential to occupational activities, sports and activities of daily living, underscoring the importance of a proper evaluation of wrist flexion and extension. Each examiner performed three measurements with a three-second interval between readings. The order of the examiners and movements was randomized using the Random Number Generator Pro $2.17^{\odot}$ program.

\section{Statistical analysis}

The Shapiro-Wilk test and Levene's test were used to determine the normality and equality of variances of the data, respectively. Based on the results of these tests, a parametric statistical method was employed. The comparison between assessment tools was performed using the paired t-test, with the level of significance set to $5 \%(p<.050)$.

Inter-examiner and intra-examiner reliability was determined based on the calculation of the intraclass correlation coefficient (ICC), with a $5 \%$ level of significance $(p<.050)$ and the calculation of $95 \%$ confidence intervals. The interpretation of the ICC was based on Fleiss ${ }^{(14)}:<0.4=$ low reliability; 0.4 to $0.75=$ moderate to good reliability; and $>0.75=$ excellent reliability. Pearson's correlation coefficients ( $r$ ) were also calculated and interpreted based on Rothstein ${ }^{(15)}: 0.90$ to 0.99 = high correlation; 0.80 to $0.89=$ good correlation; 0.70 to 0.79 = small correlation; and $\leq 0.69=$ low correlation.

The coefficient of determination $\left(R^{2}\right)$ was used as a measure of the proportion of variability in one variable that is explained by the variability in another variable, demonstrating the predictive power of the associated regression line. $R^{2}$ values range from 0 to 1 , with values closer to one indicating that the proposed model is adequate for describing a giver phenomenon $^{(16)}$ 
Bland-Altman ${ }^{(17)}$ agreement analysis was also employed. Measures were considered to be in agreement when the bias (difference between measures) was less than 10, which is considered the minimum measurable difference that can have a clinically relevant impact ${ }^{(18)}$, with a $p$-value $>.050$ and when $95 \%$ of the measures of the subjects were within the upper and lower limits of agreement ${ }^{(19,20)}$.

\section{RESULTS}

Table 1 displays the data on intra-examiner reliability for the angular measurements of flexion and extension of the left wrist by the three examiners on two different days. The paired t-test revealed no significant differences for any of the examiners ( $p>0.05$ ). Intra-examiner reliability regarding mean ROM of the left wrist was considered excellent (intraclass correlation coefficient [ICC] >0.75) for all examiners $(p<0.0001)$. Pearson's correlation coefficient indicated a high intra-examiner correlation ( $r>0.90 ; p<0.0001)$, with a mean coefficient of determination of 0.84 , meaning that $84 \%$ of the degree of wrist flexion measured on the first day was explained by the variable analyzed on the second day. For wrist extension, the coefficient of determination was 0.83 .

Table 1. Intra-examiner analysis of angles (degrees) of left and right wrists by three examiners $(A 1, A 2, A 3)$ on different days using smartphone application.

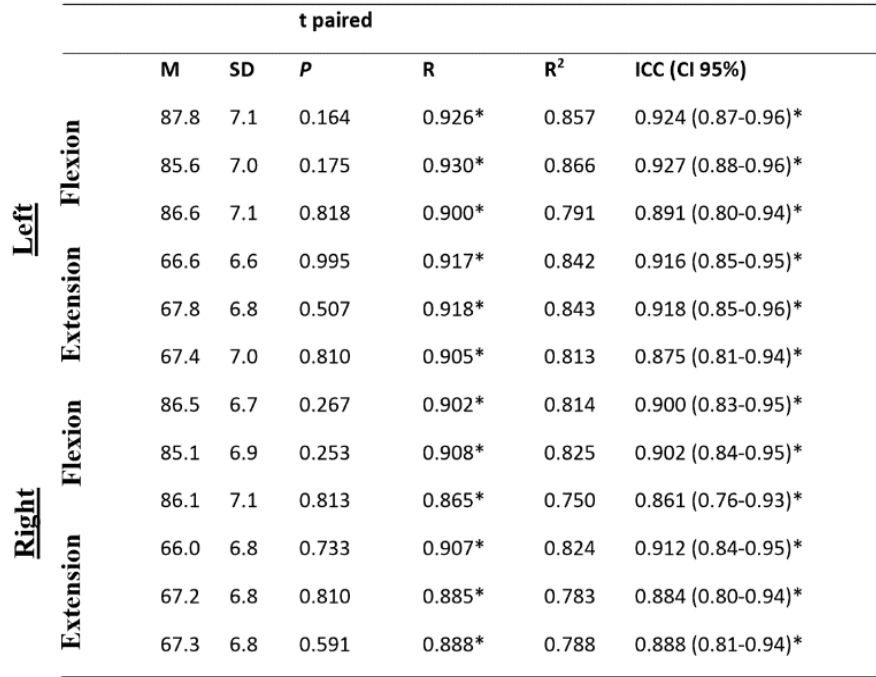

*Note: $A 1=$ Examiner $1 ; A 2=$ Examiner $2 ; A 3=$ Examiner $3 . \mathrm{M}$ : mean of two days; SD: standard deviation; $r$ : Pearson's correlation coefficient; $R^{2}$ : coefficient of determination; ICC: intraclass correlation coefficient; $\mathrm{Cl}(95 \%)$ : $95 \%$ confidence interval, $* p<0.0001$.

Regarding the mean ROM of the right wrist, intraexaminer reliability was considered excellent (ICC $>0.75$ ) for all examiners $(p<0.0001)$. Pearson's correlation coefficient indicated a high intra-examiner correlation $(r>0.80 ; p<0.0001)$, with a mean coefficient of determination of 0.79 for wrist flexion and extension.

The Bland-Altman analysis of intra-examiner measurements (Table 2) revealed agreement on all evaluations. The bias was close to zero in all evaluations, as demonstrated by the non-significant p-value (>0.05).
Regarding the limits of agreement, nearly all evaluations had data less than $5 \%$ (data not shown in table). The only exception was examiner 1 for flexion of the left wrist, with $6.9 \%$ of the data exceeding the $5 \%$ limit of agreement.

Table 2. Bias values (mean difference) and upper and lower limits of agreement for intra-examiner measurements.

\begin{tabular}{|c|c|c|c|c|c|c|}
\hline & & & Bias & ULA & LLA & $P$ \\
\hline \multirow{7}{*}{ Left } & \multirow{4}{*}{$\begin{array}{l}\frac{5}{\frac{\partial}{x}} \\
\frac{\mathrm{d}}{4}\end{array}$} & A1 & -0.6 & 4.7 & -5.9 & 0.16 \\
\hline & & $A 2$ & -0.6 & 4.7 & -5.8 & 0.17 \\
\hline & & АЗ & 0.1 & 6.7 & -6.5 & 0.82 \\
\hline & & A1 & -0.0 & 5.2 & -5.2 & 0.99 \\
\hline & \multirow{3}{*}{ 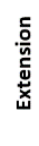 } & $A 2$ & 0.3 & 5.7 & -5.1 & 0.51 \\
\hline & & АЗ & -0.1 & 6.3 & -6.5 & 0.81 \\
\hline & & A1 & -0.5 & 5.3 & -6.3 & 0.27 \\
\hline \multirow{5}{*}{ Right } & \multirow{3}{*}{ 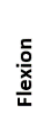 } & $A 2$ & -0.5 & 5.3 & -6.3 & 0.25 \\
\hline & & A3 & 0.1 & 7.4 & -7.2 & 0.81 \\
\hline & & A1 & 0.2 & 5.9 & -5.6 & 0.73 \\
\hline & \multirow{2}{*}{ 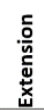 } & $A 2$ & -0.1 & 6.3 & -6.5 & 0.81 \\
\hline & & A3 & -0.3 & 6.1 & -6.6 & 0.59 \\
\hline
\end{tabular}

*Note: Bias: mean difference between measurements; ULA: upper limit of agreement (bias + 1.96*SD); LLA: lower limit of agreement (bias-1.96*SD); * $\mathrm{p}<0.05$ compared to zero (0).

Table 3 shows the results of the inter-examiner analysis of the angular measurements of flexion and extension of the left and right wrist. The paired-t test revealed no significant differences among the examiners ( $p>0.05)$. Inter-examiner reliability was considered excellent $(I C C>0.90)$ among all examiners $(p<0.0001)$. Pearson's correlation coefficient indicated a good inter-examiner correlation ( $r>0.80$; $p<0.0001$ ), with a mean coefficient of determination of 0.83 and 0.85 for the left and right wrist, respectively.

Table 3. Inter-examiner analysis of angles (degrees) of flexion and extension of left and right wrists by three examiners (A1, A2, A3) using smartphone application.

\begin{tabular}{|c|c|c|c|c|c|c|c|c|}
\hline & & & & & t paire & & & \\
\hline \multirow{7}{*}{ 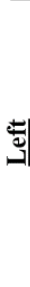 } & \multirow{5}{*}{ 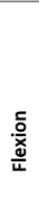 } & & M & SD & $P$ & $\mathbf{R}$ & $\mathrm{R}^{2}$ & ICC (CI 95\%) \\
\hline & & A1-A2 & 86.7 & 7.0 & 0.282 & $0.933 *$ & 0.870 & $0.904(0.88-0.96)^{*}$ \\
\hline & & A1-A3 & 87.3 & 7.1 & 0.557 & $0.858^{*}$ & 0.736 & $0.862(0.75-0.92)^{*}$ \\
\hline & & $A 2-A 3$ & 86.6 & 7.0 & 0.399 & $0.886^{*}$ & 0.785 & $0.875(0.80-0.94)^{*}$ \\
\hline & & A1-A2 & 67.3 & 6.7 & 0.363 & $0.930 *$ & 0.865 & $0.910(0.87-0.96)^{*}$ \\
\hline & \multirow{3}{*}{ 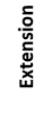 } & A1-A3 & 67.0 & 6.7 & 0.557 & $0.941 *$ & 0.887 & $0.934(0.89-0.97)^{*}$ \\
\hline & & A2-A3 & 67.7 & 6.7 & 0.399 & $0.915^{*}$ & 0.837 & $0.913(0.85-0.95)^{*}$ \\
\hline \multirow{6}{*}{ 혬 } & & A1-A2 & 85.4 & 6.8 & 0.305 & $0.934 *$ & 0.872 & $0.917(0.88-0.96)^{*}$ \\
\hline & \multirow{3}{*}{ 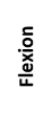 } & A1-A3 & 86.2 & 7.0 & 0.810 & $0.926 *$ & 0.857 & $0.928(0.87-0.96)^{*}$ \\
\hline & & A2-A3 & 85.5 & 7.1 & 0.352 & $0.920 *$ & 0.845 & $0.902(0.86-0.96)^{*}$ \\
\hline & & A1-A2 & 66.6 & 6.9 & 0.467 & $0.916^{*}$ & 0.840 & $0.905(0.85-0.95)^{*}$ \\
\hline & \multirow{2}{*}{ 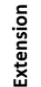 } & A1-A3 & 66.5 & 6.9 & 0.451 & $0.929 *$ & 0.862 & $0.915(0.87-0.96)^{*}$ \\
\hline & & A2-A3 & 67.2 & 7.0 & 0.936 & $0.893^{*}$ & 0.800 & $0.885(0.81-0.94)^{*}$ \\
\hline
\end{tabular}

${ }^{*}$ Note: $\mathrm{A} 1=$ Examiner $1 ; \mathrm{A} 2=$ Examiner $2 ; \mathrm{A} 3=$ Examiner $3 . \mathrm{M}$ : mean among examiners; SD: standard deviation; $r$ : Pearson's correlation coefficient; $R^{2}$ : coefficient of determination; ICC: intraclass correlation coefficient; $\mathrm{Cl}(95 \%)$ : $95 \%$ confidence interval, ${ }^{*} p<0.0001$. 
Table 4. Bias values (mean difference) and upper and lower limits of agreement for inter-examiner measurements.

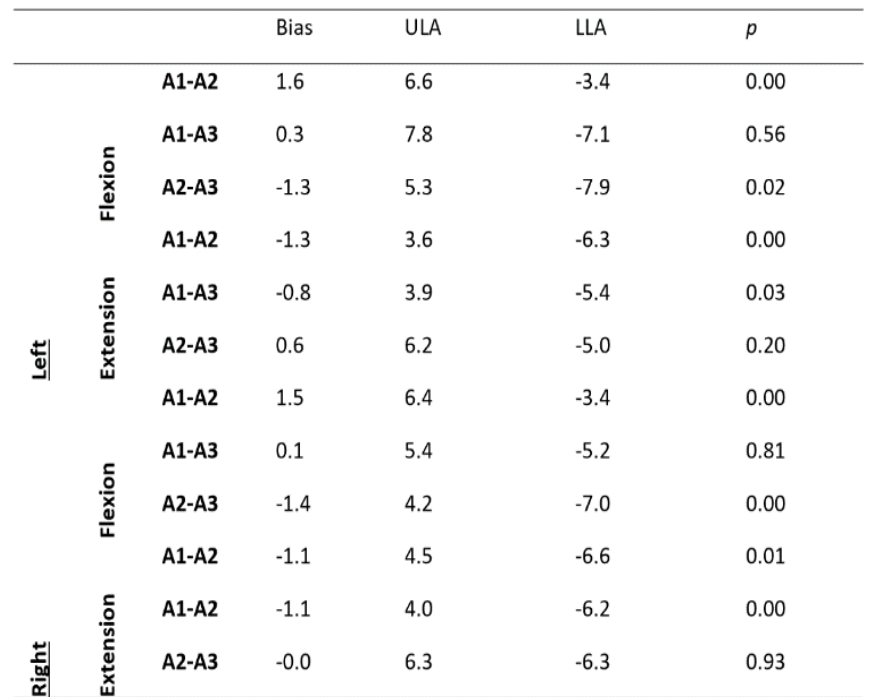

${ }^{*}$ Note: Bias: mean difference between measurements; ULA: upper limit of agreement (bias + 1.96*SD); LLA: lower limit of agreement (bias-1.96*SD); * $p<0.05$ compared to zero (0).

\section{DISCUSSION}

The present study evaluated inter-examiner and interexaminer reliability of the Goniometer $P R O^{\odot}$ smartphone application regarding the measurement of wrist angles in healthy young women. Three independent trained examiners performed the evaluations. The results revealed that the goniometric smartphone application exhibited excellent intraexaminer and inter-examiner reliability.

The mean difference was only one degree for all evaluations in both the intra-examiner and inter-examiner analyses. According to Hambly et al. ${ }^{(21)}$, small differences in angular measurements of ROM do not have a relevant clinical impact. Jakobsen et al. ${ }^{(18)}$ determined that 10 degrees is the minimum difference to be considered a relevant measurement error.

The agreement analysis proposed by Bland and Altman in $1986^{(17)}$, is currently considered the preferable manner for evaluating agreement in the results of measurements, establishing the limits of agreement and determining the mean difference (bias) ${ }^{(19,20)}$. Correlation analyses quantify the degree with which two variables are associated, but often overestimate the results ${ }^{(16)}$.

The present findings revealed better agreement in the intra-examiner analysis, with a lower bias than that initially defined $\left(10^{\circ}\right)$, with a variation ranging from 0.0 to $0.6^{\circ}$. Only examiner 1 exceeded the limits of agreement by $6.9 \%$ in the evaluation of left wrist flexion.

In the inter-examiner analysis, eight of the 12 evaluations had a bias somewhat more distant from zero, ranging from 0.8 to $1.6^{\circ}$, with less agreement on these evaluations. Santos et al. ${ }^{(22)}$ report similar findings, with the inter-examiner evaluation presenting a bias distanced from zero and lower agreement among examiners, but the differences did not exceed three degrees.

The inter-examiner reliability analyses presented better results compared to the intra-examiner analyses. This may be explained by the fact that the examiners were trained together, leading to similar evaluations. Thus, goniometric applications are reliable for evaluations by different examiners $^{(23,24,25)}$.

Examining healthy individuals, Pourahmad et al. ${ }^{(13)}$ concluded that goniometric smartphone applications are reliable for determining wrist angles, with high intra-examiner $(I C C>0.83)$ and inter-examiner (ICC $>0.79)$ reliability. The excellent intra-examiner reliability found in the study may be explained by the attachment of the smartphone to the wrist. In contrast, only the distal third of the forearm was secured in the present investigation, in which better inter-examiner reliability $(I C C>0.90)$ and lower intra-examiner reliability (ICC $>0.75$ ) were found.

The data used for the inter-examiner analysis were collected only on the first day, as the results were similar between the first and second day, with no large differences.

Several studies have investigated the reliability of smartphone applications for the measurement of active and passive ROM in different joints. Otteret al. ${ }^{(26)}$ analyzed the passive movement of the ankle using the Dr. Goniometer ${ }^{\mathcal{O}}$ application for Iphone ${ }^{\odot}$, reporting excellent inter-examiner $($ ICC $=0.786$ ) and intra-examiner (ICC $=0.875$ ) reliability. Other researchers found high intra-examiner and inter-examiner reliability (ICC = 0.998) in the measurement of the ROM of the elbow using the same application (Dr. Goniometer ${ }^{\odot}$ for Iphone ${ }^{\odot}$ ). Reliability analyses have also been performed on other joints, with studies reporting excellent intra-examiner and interexaminer reliability in measurements of shoulder ROM in different positions using the Clinometer ${ }^{\odot}$ application $^{(6,27)}$.

Kuegler et al. ${ }^{(28)}$ compared 15 applications for two operational systems to evaluate the reliability and validity of these applications regarding ROM in individuals in the postoperative period following hand surgery. Fourteen applications proved to be reliable and valid, but, according to the literature, these applications are not used in clinical practice.

Another aspect to consider is the comparison between experienced and inexperienced examiners ${ }^{(3)}$. Previous studies have investigated the level of agreement of inexperienced examiners regarding knee flexion in healthy subjects ${ }^{(20)}$ compared to the evaluation of experienced examiners for the determination of the reliability and validity of goniometric applications $^{(29)}$.

The present study has two important limitations to consider: 1) the possible movement of the smartphone on the participant's skin and 2) the attempts of some participants to compensate the wrist movement by lifting the forearm off the table. However, measurement bias was minimized by strapping the forearm to the table, as described above. Moreover, the results can only be extrapolated to healthy young women and not to other populations. 
Future studies should analyze the reliability of smartphone applications on 1) different joints of the body, 2) in patients with different adverse health conditions, 3) using different goniometric applications (software), 4) using different brands of telephone (hardware), 5) using equipment with shorter and longer times of use and 6) comparing the evaluations of experienced and inexperienced examiners. Such studies could contribute to the clarification of issues that remain in the literature on this topic.

\section{CONCLUSION}

Considering the procedures used in the present study, the Goniometer $P R O^{\odot}$ goniometric smartphone application can be used in clinical practice for the evaluation of the range of motion of the wrists of women, as demonstrated by the excellent intra-examiner and inter-examiner reliability and good level of agreement.

AUTHORS' CONTRIBUTION: MSBS and TW developed the study and the corresponding text, AMP performed the data collection; LR carried out the supervision and orientation of the study; LABF and WMP performed a critical intellectual review of the manuscript. All authors read and approved the final manuscript.

FINANCIAL SUPPORT: Nothing to declare.

CONFLICT OF INTEREST: The authors declare that they have no conflict of interest.

\section{REFERENCES}

1. Milani P, Coccetta CA, Rabini A, Sciarra T, Massazza G, Ferriero G. Mobile Smartphone Applications for Body Position Measurement in Rehabilitation: A Review of Goniometric Tools. PM \& R. 2014;6(11):1038-1043.

2. Jenny JY. Measurement of the knee flexion angle with a Smartphone-application is precise and accurate. J Arthroplasty. 2013;28(5):784-7.

3. Milanese S, Gordon S, Buettner P, Flavell C, Ruston S, Cor $D$, et al. Reliability and concurrent validity of knee angle measurement: Smart phone App versus Universal Goniometer used by experienced and novice clinicians. Manual Therapy. 2014;19(6):56974.

4. Mitchell K, Gutierrez SB, Sutton S, Morton S, Morgenthaler A. Reliability and validity of goniometric iPhone applications for the assessment of active shoulder external rotation. Physiother Theory Pract. 2014;30(7):521-525.

5. Conte ALF, Marques AP, Casarotto RA, João SMA. Handedness influences passive shoulder range of motion in non athlete adult women: J of Manipulative and Physiological Therapeutics Handedness in Shoulder Range Motion. 2009;32(2):149-53.

6. Shin SH, Ro DH, Lee OS, Oh JH, Kim SH. Within-day reliability of shoulder range of motion measurement with a smartphone. Man Ther. 2012;17(4):298-304.
7. Hancock GE, Hepworth T, Wembridge K. Accuracy and reliability of knee goniometry methods. J Exp Orthp. 2018;5(1):46.

8. Souza AC, Alexandre NMC, Guirardello EB, Psychometric properties in instruments evaluation of reliability and validity. Brasil Epidemiol Serv Saude. 2017;26(3):649-59.

9. Bolarinwa OA, Principles and Methods of Validity and Reliability Testing of Questionnaires Used in Social and Health Science Researches. Niger Postgrad Med J. 2015;22(4):195-201.

10. Magee DJ. Orthopedic Physical Assessment. 6th ed. St. Louis, Missouri: Elsevier Health Sciences, 2014.

11. Carter TI, Pansy B, Wolff AL. Accuracy and reliability of three different techniques for manual goniometry for wrist motion: a cadaveric study. J Hand Surg. 2009;34(8):1422-8.

12. Gehrmann S, Roeger T, Kaufmann R, Schaedle A, Lögters $\mathrm{T}$, Windolf $\mathrm{J}$. Wrist motion analysis in scaphoid nonunion. Eur J Trauma Emerg Surg. 2016; 42(1):11-14.

13. Pourahmad MR, Takamjani IE, Sarrafzadeh J, et al. Reliability and concurrent validity of a new iPhone ${ }^{\circledR}$ goniometric application for measuring active wrist range of motion: a cross-sectional study in asymptomatic subjects. J. Anat. 2017;230(3):484495.

14. Fleiss JL, Levin B, Paik MC. Statistical methods for rates and proportions. 3thed: John Wiley \& Sons, 2003.

15. Rothstein JM, Miller PJ, Roettger RF. Goniometric reliability in a clinical setting. Elbow and knee measurements. Journal Phys Ther. 1983;63(10):16115.

16. Field A. Discovering Statistics Using SPSS. Sage publications. Thousand Oaks. 2009.

17. Altman DG, Bland JM. Measurement in medicine: the analysis of method comparison studies. Statistician. 1983;32(3):307-17.

18. Jakobsen TL, Christensen M, Christensen SS, Olsen $\mathrm{M}$, Bandholm T. Reliability of knee joint range of motion and circumference measurements after total knee arthroplasty: does tester experience matter? Physiother Res Int. 2010;15(3):126-134.

19. Giavarina D."Understanding Bland Altman Analysis. Biochem Med. 2015;25(2):141-151.

20. Hirakata VN, Camey AS. Bland-altman analysis of agreement between methods. Rev HCPA. 2009; 29(3):261-268.

21. Hambly K, Sibley $\mathrm{R}$, Ockendon M. Agreement between a novel smartphone application and a long arm goniometer for assessment of active knee flexion by an inexperienced tester. J Physiother Rehabil. 2012;2:1-14.

22. Santos CA, Pauchard NB, Guilloteau AC. Reliability assessment of measuring active wrist pronation and supination range of motion with a smartphone. Hand Surg Rehabil. 2017;36(5):338-345. 
23. Ferriero G, Sartorio F, Foti C, Primavera D, Brigatti E, Vercelli S. Reliability of a new application for smartphones ( $\mathrm{Dr}$ Goniometer) for elbow angle measurement. PM \& R. 2011;3(12):1153-4.

24. Ferriero G, Vercelli S, Sartorio F, Lasa MS, llieva E, Brigatti $E$, et al. Reliability of a smartphone-based goniometer for knee joint goniometry. Int J Rehabil Res. 2013;36(2):146-51.

25. Blonna D, Zarkadas PC, Fitzsimmons JS, O'Driscoll SW. Validation of a photography-based goniometry method for measuring joint range of motion. J Shoulder Elbow Surg. 2012;21(1):29-35.

26. Otter SJ, Agalliu B, Baer N, Hales G, Harvey K, James $\mathrm{K}$, et al. The reliability of a smartphone goniometer application compared with a traditional goniometer for measuring first metatarsophalangeal joint dorsiflexion. $J$ of foot and ankle research. 2015;8(1):1-7.

27. Alba-Martín R. Reliability and validity of shoulder and elbow measurements: Analysis of an android application and a goniometer. Rehabilitación. 2016;50(2):71-74.

28. Kuegler $P$, Wurzer $P$, Tuca A, Sendlhofer G, Lumenta $\mathrm{DB}$, Giretzlehner $\mathrm{M}$, et al. Goniometer-apps in hand surgery and their applicability in daily clinical practice. Safety in Health. 2015; 1-11.

29. WellmonRH, Gulick TD, Paterson ML, Gulick CN. Validity and Reliability of 2 Goniometric Mobile Apps: Device, Application, and Examiner Factors. J of Sport Rehabilitation. 2016;25(4):371-379. 